\title{
Magnetic Resonance Imaging of an Area of Interest with and without Contrast
}

National Cancer Institute

\section{Source}

National Cancer Institute. Magnetic Resonance Imaging of an Area of Interest with and

without Contrast. NCl Thesaurus. Code C137919.

Magnetic resonance imaging of an anatomical area of interest with and without a

contrast agent to enhance the image. 